\title{
AN EQUAL-ORDER FINITE ELEMENT FRAMEWORK FOR INCOMPRESSIBLE NON-NEWTONIAN FLOW PROBLEMS
}

\author{
DOUGLAS R. Q. PACHECO ${ }^{1}$ AND OLAF STEINBACH ${ }^{2}$ \\ ${ }^{1,2}$ Institute of Applied Mathematics, Graz University of Technology \\ Steyrergasse 30/III, 8010 Graz, Austria \\ pacheco@math.tugraz.at
}

Key words: Non-Newtonian fluids, Incompressible flows, Stabilised finite element methods, Residualbased stabilisation, Pressure boundary conditions, Hemodynamics

\begin{abstract}
Various materials of engineering and biomedical interest can be modelled as generalised Newtonian fluids, i.e., via an apparent viscosity depending locally on the flow field. In spite of the particular features of those models, they are often handled in practice by classical numerical techniques originally conceived for Newtonian fluids. Methods designed specifically for the generalised case are rather scarce in the literature, as well as their use in practical applications. As it turns out, tackling nonNewtonian problems with standard finite element formulations can have undesired consequences such as the induction of spurious pressure boundary layers and the emergence of natural boundary conditions not suitable for realistic flow scenarios. In this context, we introduce a novel framework that deals with those issues while maintaining simplicity and low computational cost. The new stabilised formulation is based on a modified system combining the continuity equation with a Poisson equation for the pressure and consistent pressure boundary conditions. A weak enforcement of the rheological law is employed to enable full consistency even for first-order finite element pairs. Simple numerical examples are provided to demonstrate the potential of our method in yielding accurate solutions for relevant problems.
\end{abstract}

\section{INTRODUCTION}

Although blood can normally be considered as a fluid, it is not a liquid, but an organic tissue consisting of living and non-living components immersed in a liquid matrix. Therefore, modelling its behaviour mathematically is by no means a trivial task. In most arteries of healthy individuals, blood flows basically as a Newtonian fluid with uniform viscosity and density. In low-shear regions such as certain veins, small arteries or aneurysms, non-Newtonian effects like shear thinning and viscoelasticity can play a major role in hemodynamics [1]. The non-Newtonian behaviour most frequently observed in blood flows is shear thinning, characterised by decreasing apparent viscosity in the presence of increasing shear rates. This is mostly due to the three-dimensional structures formed by the aggregation of red blood cells [1]. Since these structures take several seconds to form and cannot withstand high shear rates, this non-Newtonian response is seen mainly in flow regions experiencing low shear rates stably throughout time [2].

In particular, our focus is placed on generalised Newtonian models. This popular approach allows for local variations of blood's effective viscosity depending on shear rate levels. It is capable of accounting for non-Newtonian phenomena such as shear thinning and plug flow, while maintaining a similar mathemat- 
ical and computational framework as used for Newtonian fluids. In fact, several simple models provide excellent fits to experimental data [1,3]. Such generalised models, sometimes called quasi-Newtonian, are used not only in hemodynamics but also in polymeric flow simulations of industrial interest. Due to the apparent similarity between Newtonian and quasi-Newtonian equations, one is easily tempted to try solving the generalised problem using classical numerical methods. Although it is often possible to do so by applying certain modifications, that is neither physically nor mathematically ideal. Yet, before commenting on the limitations of the existing methods, we shall briefly outline some typical numerical issues encountered in incompressible flow problems.

When using finite element methods for the approximation of incompressible flows, one must carefully choose velocity and pressure basis functions. Using the same polynomial order for both quantities, for instance, violates the famous Ladyzhenskaya-Babuška-Brezzi (LBB) condition, which leads to unstable methods. However, since the findings of Hughes and co-workers four decades ago [4, 5], it has become standard to employ stabilisation methods such as PSPG or GLS to circumvent the LBB condition and allow equal-order shape functions for all unknowns. Nonetheless, most stabilisation methods are based on perturbed incompressibility equations devised under classical Newtonian assumptions. Probably for that reason, the use of LBB-compatible finite elements is somewhat more frequent in the literature for quasi-Newtonian fluids [6-16]. Although such compatible spaces offer an ideal setting from a theoretical standpoint, they are not always viable options. There is thus great practical appeal for first-order elements - especially in biomedical applications, where higher-order meshes are rarely available.

There are a handful of stabilisation methods which are not residual-based and can therefore be applied

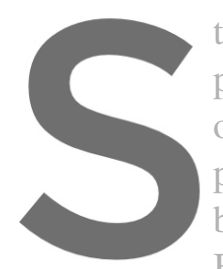

to generalised Newtoni

penalty method relaxing i

often for quasi-Newtonian

pressure approximation

by Knauf et al. [22] to a

Bocher [23] was used strcesstur
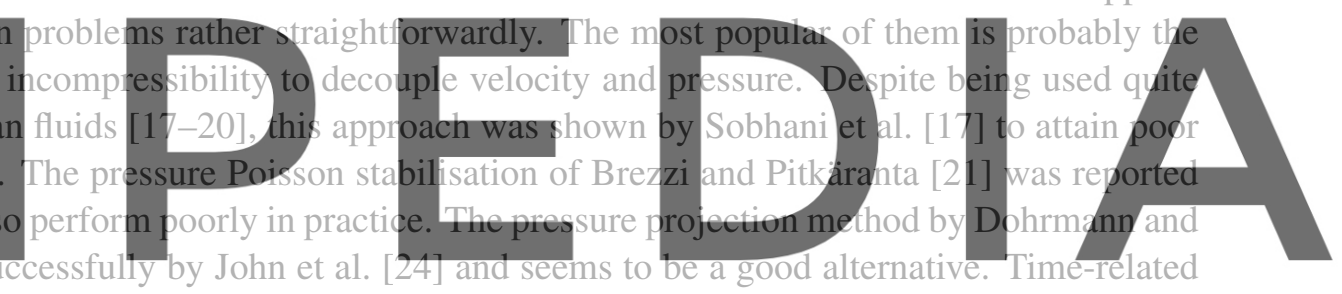

methods which can be applied straightforwardly to the quasi-Newtonian case are artificial compressibility

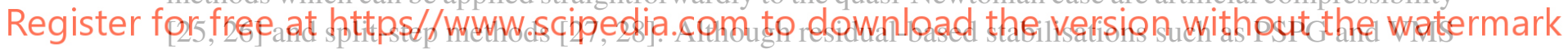

can also be used [29-32], their loss of consistency for linear elements can yield inaccurate results, as discussed in Subsection 3.1 .

To the best of our knowledge, there are very few works in the literature dedicated to designing stabilisation techniques for generalised Newtonian problems. Some of them require introducing the viscous stress as an additional tensor-valued unknown [33,34], leading to prohibitive computational costs in practical applications. The variational muti-scale (VMS) approach proposed by Masud and Kwack [30] offers the efficiency and simplicity of residual-based methods, but can induce spurious pressure boundary layers for low-order discretisations. With these challenges in mind, this work presents, as its main contribution, a new residual-based framework for equal-order finite element approximations of quasi-Newtonian problems. The most important feature of our new method is overcoming the loss of consistency of standard PSPG-like stabilisation terms in the lowest-order case, by augmenting the continuity equation with a pressure Poisson equation $[35,36]$ with consistent boundary conditions. In doing so, we achieve considerable improvements in robustness and accuracy with respect to state-of-the-art residual-based stabilisations. 


\section{PRELIMINARIES}

The balance of linear momentum and mass for a stationary incompressible flow of a generalised Newtonian fluid can be stated as, respectively,

$$
\begin{aligned}
(\rho \nabla \mathbf{u}) \mathbf{u}-\nabla \cdot\left(2 \mu \nabla^{s} \mathbf{u}\right)+\nabla p & =\mathbf{0} \\
\nabla \cdot \mathbf{u} & =0
\end{aligned}
$$

where $\mathbf{u}$ is the flow velocity, $p$ is the pressure, $\rho$ is the fluid's density, $\mu$ is the (apparent) dynamic viscosity and $\nabla^{s} \mathbf{u}$ is the symmetric part of the velocity gradient, namely,

$$
\nabla^{s} \mathbf{u}:=\frac{1}{2}\left[\nabla \mathbf{u}+(\nabla \mathbf{u})^{\top}\right] .
$$

In hemodynamic and polymeric flows, the viscosity is normally modelled through a nonlinear depen-

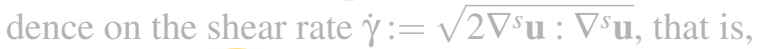

$$
\mu=\mu\left(\nabla^{s} \mathbf{u}\right)=\eta\left(\dot{\gamma}\left(\nabla^{s} \mathbf{u}\right)\right) .
$$

Before introducing the variational formulations, let us consider a spatial domain $\Omega \subset \mathbb{R}^{d}, d=2,3$, with a Lipschitz boundary $\Gamma:=\partial \Omega$ decomposed into two non-overlapping regions $\Gamma_{D}$ and $\Gamma_{N}$. In $\Gamma_{D}$ the velocities are prescribed: $\left.\mathbf{u}\right|_{\Gamma_{D}}=\mathrm{g}$. The kind of data prescribed on $\Gamma_{N}$ depends on the weak form used,

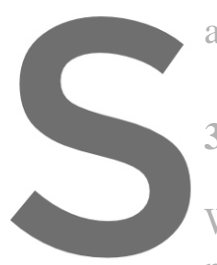
as will be discussed later on.

\section{STABILISED FORMULATIONS}

We now present the most classical residual-based
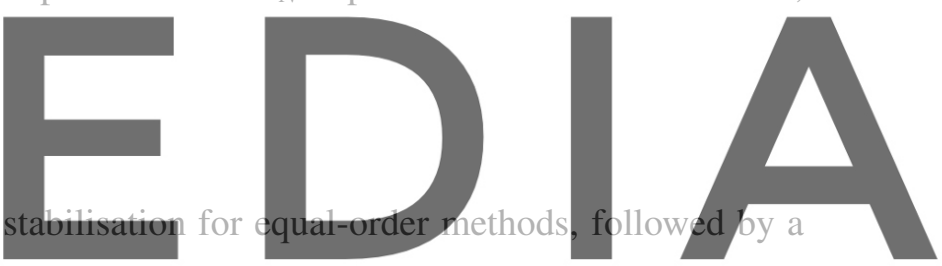

\section{Register for free at https//www.scipedia.com to download the version without the watermark}

The pressure-stabilised Petrov-Galerkin (PSPG) method is the most popular stabilisation approach for equal-order Navier-Stokes solvers. It consists of relaxing the incompressibility constraint with a (strong) weighted residual of the momentum equation. The discrete variational formulation is to find $\left(\mathbf{u}_{h}, p_{h}\right) \in$ $\left[X_{h}\right]^{d} \times X_{h}$, with $\left.\mathbf{u}_{h}\right|_{\Gamma_{D}}=\mathbf{g}_{h}$, such that for all $\left(\mathbf{w}_{h}, q_{h}\right) \in\left[X_{h}\right]^{d} \times X_{h}$, with $\left.\mathbf{w}\right|_{\Gamma_{D}}=\mathbf{0}$,

$$
\begin{aligned}
\left\langle\mathbf{w}_{h},\left(\rho \nabla \mathbf{u}_{h}\right) \mathbf{u}_{h}\right\rangle_{\Omega}+\left\langle\nabla^{s} \mathbf{w}_{h}, 2 \mu\left(\nabla^{s} \mathbf{u}_{h}\right) \nabla^{s} \mathbf{u}_{h}\right\rangle_{\Omega}-\left\langle\nabla \cdot \mathbf{w}_{h}, p_{h}\right\rangle_{\Omega} & =\left\langle\mathbf{w}_{h}, \mathbf{t}\right\rangle_{\Gamma_{N}}, \\
\left\langle q_{h}, \nabla \cdot \mathbf{u}_{h}\right\rangle_{\Omega}+\sum_{e=1}^{N_{e}}\left\langle\frac{\alpha h_{e}{ }^{2}}{\mu\left(\nabla^{s} \mathbf{u}_{h}\right)} \nabla q_{h}, \nabla p_{h}-\nabla \cdot\left[2 \mu\left(\nabla^{s} \mathbf{u}\right) \nabla^{s} \mathbf{u}\right]+\left(\rho \nabla \mathbf{u}_{h}\right) \mathbf{u}_{h}\right\rangle_{\Omega_{e}} & =0,
\end{aligned}
$$

where $X_{h}$ is a continuous finite element space, $\mathbf{t}:=\left[2 \mu\left(\nabla^{s} \mathbf{u}\right) \nabla^{s} \mathbf{u}\right] \mathbf{n}-p \mathbf{n}, N_{e}$ is the number of elements $\Omega_{e}$, $\alpha$ is a positive parameter and $\mathbf{n}$ is the outward unit normal vector on $\Gamma$. Let us also define $h=\max \left\{h_{e}\right\}$. Using the stabilisation parameter as $O\left(h_{e}{ }^{2}\right)$, as done above, is the optimal choice for diffusion-dominated flows [37]. For $\alpha$ sufficiently large, the system is stable for equal-order pairs due to the bilinear form $\left\langle\nabla q_{h}, \nabla p_{h}\right\rangle_{\Omega}$, which breaks the saddle-point structure of the problem and the LBB requirement. The 
idea behind including the remaining terms of the residual is to render the perturbation to the continuity equation smaller where the solution is already accurate enough. This is very important for the accuracy and quality of the approximations [38, 39], and here arises the issue of using PSPG with linear elements. In that case, and when using simplicial elements, the velocity gradient is piecewise constant and, consequently, so is the (discrete) viscous stress tensor $\mathbb{S}_{h}$. Hence:

$$
\sum_{e=1}^{N_{e}}\left\langle\frac{\alpha h_{e}^{2}}{\mu\left(\nabla^{s} \mathbf{u}_{h}\right)} \nabla q_{h}, \nabla \cdot \mathbb{S}_{h}\right\rangle_{\Omega_{e}}=\sum_{e=1}^{N_{e}}\left\langle\frac{\alpha h_{e}^{2}}{\mu\left(\nabla^{s} \mathbf{u}_{h}\right)} \nabla q_{h}, \mathbf{0}\right\rangle_{\Omega_{e}}=0
$$

that is, we completely lose the viscous part of the residual. This means that, no matter how fine the mesh is, the added residual will never vanish. An incomplete residual can spoil coarse grid accuracy and restrict the choice of the stabilisation parameter. As a matter of fact, it can be shown that an "inviscid" residual induces so-called spurious pressure boundary layers [40]. Although remedies exist in the Newtonian case $[38,39,41]$, they cannot be directly applied to fluids with shear-dependent viscosity.

\section{Another shortcoming of the standard formulation (3) is its natural boundary conditions (BCs) given in terms of tractions $\mathbf{t}$. Although very often done [24], setting $\mathbf{t}=\mathbf{0}$ on open boundaries leads to spurious velocity and pressure oscillations [42, 43]. For constant viscosity, there is a simple remedy: using the Laplacian form of the stress-divergence, that is,}
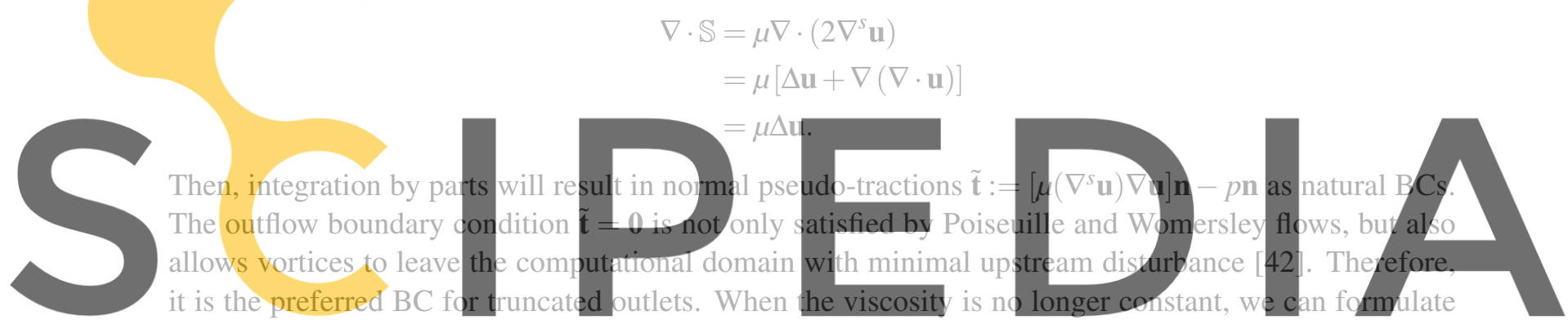

the problem in a generalised Laplacian form by writing

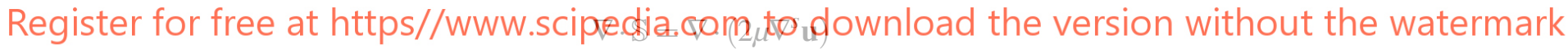

$$
\begin{aligned}
& =\mu[\Delta \mathbf{u}+\nabla(\nabla \cdot \mathbf{u})]+2 \nabla^{s} \mathbf{u} \nabla \mu \\
& =\mu \Delta \mathbf{u}+2 \nabla^{s} \mathbf{u} \nabla \mu,
\end{aligned}
$$

and using integration by parts only on the Laplacian term $\mu \Delta \mathbf{u}$. The weak form of the momentum equation then becomes [44]

$$
\left\langle\mathbf{w}_{h},\left(\rho \nabla \mathbf{u}_{h}\right) \mathbf{u}_{h}\right\rangle_{\Omega}+\left\langle\nabla \mathbf{w}_{h}, \mu_{h} \nabla \mathbf{u}_{h}\right\rangle_{\Omega}-\left\langle\mathbf{w}_{h},\left(\nabla \mathbf{u}_{h}\right)^{\top} \nabla \mu_{h}\right\rangle_{\Omega}-\left\langle\nabla \cdot \mathbf{w}_{h}, p_{h}\right\rangle_{\Omega}=\left\langle\mathbf{w}_{h}, \tilde{\mathbf{t}}\right\rangle_{\Gamma_{N}},
$$

where $\mu_{h}$ is a continuous projection of the viscosity field, as discussed later on.

\subsection{The generalised boundary vorticity stabilisation method}

We will next present a generalisation of the boundary vorticity stabilisation (BVS) by Pacheco et al. [45] to quasi-Newtonian problems. The BVS is a residual-based formulation containing a first-order boundary term proportional to the vorticity $\nabla \times \mathbf{u}$, which guarantees consistency even for linear elements. Details on the method for the Newtonian case can be found in our previous article [45]. 


\subsubsection{Strong form}

We start by constructing an equivalent PDE system to replace the classical momentum-mass system (1)-(2). The proposed boundary value problem (BVP) reads:

$$
\begin{aligned}
(\rho \nabla \mathbf{u}) \mathbf{u}-\mu \Delta \mathbf{u}-2 \nabla^{s} \mathbf{u} \nabla \mu+\nabla p & =\mathbf{0}, \\
-\Delta p & =\nabla \cdot\left[(\rho \nabla \mathbf{u}) \mathbf{u}-2 \nabla^{s} \mathbf{u} \nabla \mu\right]+[\nabla \times(\nabla \times \mathbf{u})] \cdot \nabla \mu-\beta \nabla \cdot \mathbf{u}, \\
\left.\frac{\partial p}{\partial n}\right|_{\Gamma} & =\mathbf{n} \cdot\left[2 \nabla^{s} \mathbf{u} \nabla \mu-(\rho \nabla \mathbf{u}) \mathbf{u}-\mu \nabla \times(\nabla \times \mathbf{u})\right],
\end{aligned}
$$

where $\beta$ is some given positive function to be defined later. The velocity BCs were omitted because they play no role in the following discussion. We wish to show that, for sufficiently regular $p$ and $\mathbf{u}$, the new system is equivalent to the classical one ((1)-(2)). The first step is to apply the divergence operator to Eq. (7), leading to

$$
\begin{aligned}
\Delta p & =\nabla \cdot\left[\mu \Delta \mathbf{u}+2 \nabla^{s} \mathbf{u} \nabla \mu-(\rho \nabla \mathbf{u}) \mathbf{u}\right] \\
& =\nabla \cdot\left[2 \nabla^{s} \mathbf{u} \nabla \mu-(\rho \nabla \mathbf{u}) \mathbf{u}\right]+\nabla \mu \cdot \Delta \mathbf{u}+\mu \nabla \cdot(\Delta \mathbf{u}),
\end{aligned}
$$

which when added to Eq. (8) gives

$$
0=[\nabla \times(\nabla \times \mathbf{u})] \cdot \nabla \mu-\beta \nabla \cdot \mathbf{u}+\nabla \mu \cdot \Delta \mathbf{u}+\mu \nabla \cdot(\Delta \mathbf{u})
$$

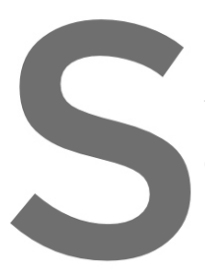

$$
=\mu \nabla \cdot(\Delta \mathbf{u})+\nabla u \cdot[\Delta \mathbf{u}-\nabla \times(\nabla \times \mathbf{u})]-\beta \nabla \cdot \mathbf{u}
$$

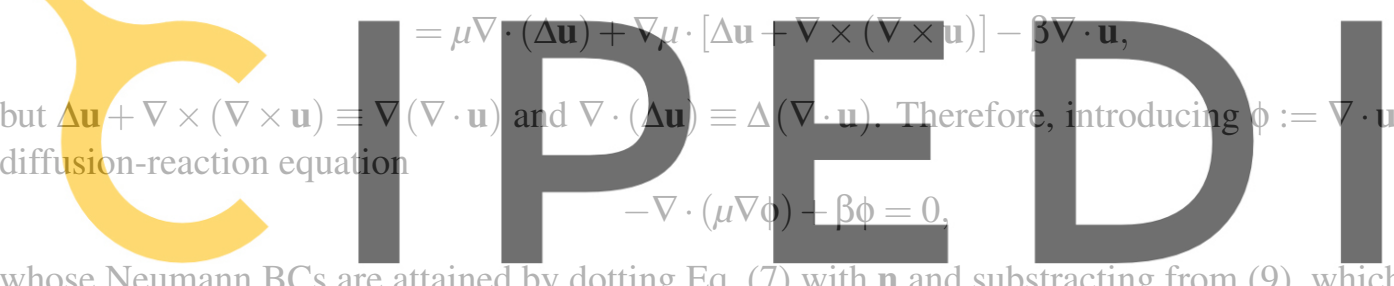

whose Neumann BCs are attained by dotting Eq. (7) with in and substracting from (9), which leads to

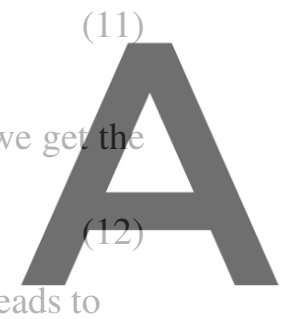

\section{Register for free at https//www.scipedie.cqmito download the version without the watermark}

$$
\begin{aligned}
& =\mathrm{n} \cdot[\nabla(\nabla \cdot \mathbf{u})] \\
& =\frac{\partial \phi}{\partial n} .
\end{aligned}
$$

The solution of Eq. (12) is thus $\phi \equiv 0$, that is, $\nabla \cdot \mathbf{u}=0$ in $\Omega$, as we wanted. Now that we have recovered Eq. (2), the equivalence between Eqs. (7) and (1) is straightforward. Proving the other direction, i.e., that the standard Navier-Stokes system implies the modified BVP is similar but simpler, so we omit this part.

\subsubsection{Weak formulation}

With the new modified system we can start deriving our stabilised formulation. Integration by parts gives

$$
\langle\nabla q, \nabla p\rangle_{\Omega}-\left\langle q, \frac{\partial p}{\partial n}\right\rangle_{\Gamma}=\left\langle q, \nabla \cdot\left[(\rho \nabla \mathbf{u}) \mathbf{u}-2 \nabla^{s} \mathbf{u} \nabla \mu\right]\right\rangle_{\Omega}+\langle q,[\nabla \times(\nabla \times \mathbf{u})] \cdot \nabla \mu-\beta \nabla \cdot \mathbf{u}\rangle_{\Omega} .
$$


Integrating the first term on the right-hand side by parts and enforcing the Neumann BC (9) yields

$$
\begin{array}{r}
\langle q, \beta \nabla \cdot \mathbf{u}\rangle_{\Omega}+\langle\nabla q, \nabla p+(\rho \nabla \mathbf{u}) \mathbf{u}\rangle_{\Omega}= \\
\left\langle\nabla q,\left[\nabla \mathbf{u}+(\nabla \mathbf{u})^{\top}\right] \nabla \mu\right\rangle_{\Omega}+\langle q,[\nabla \times(\nabla \times \mathbf{u})] \cdot \nabla \mu\rangle_{\Omega}-\langle q \mathbf{n}, \mu \nabla \times(\nabla \times \mathbf{u})\rangle_{\Gamma},
\end{array}
$$

but

$$
\begin{aligned}
\langle q \mathbf{n}, \mu \nabla \times(\nabla \times \mathbf{u})\rangle_{\Gamma} & =\langle q, \mu \overbrace{\nabla \cdot[\nabla \times}^{\equiv 0}(\nabla \times \mathbf{u})]\rangle_{\Omega}+\langle\nabla(q \mu), \nabla \times(\nabla \times \mathbf{u})\rangle_{\Omega} \\
& =\langle\nabla q, \mu \nabla \times(\nabla \times \mathbf{u})\rangle_{\Omega}+\langle q, \nabla \mu \cdot[\nabla \times(\nabla \times \mathbf{u})]\rangle_{\Omega} .
\end{aligned}
$$

Therefore, the right-hand side of Eq .(14) becomes

$$
\left\langle\nabla q,\left[\nabla \mathbf{u}+(\nabla \mathbf{u})^{\top}\right] \nabla \mu\right\rangle_{\Omega}-\langle\nabla q, \mu \nabla \times(\nabla \times \mathbf{u})\rangle_{\Omega} .
$$

As in the PSPG formulation, there is a second-order term which cannot be approximated by first-order finite element spaces. To preserve the viscous residual we must rewrite this second-order term with first-order derivatives only. Using integration by parts once again gives

$$
\langle\mu \nabla q, \nabla \times(\nabla \times \mathbf{u})\rangle_{\Omega}=\langle\nabla q \times \mathbf{n}, \mu \nabla \times \mathbf{u}\rangle_{\Gamma}+\langle\nabla \times(\mu \nabla q), \nabla \times \mathbf{u}\rangle_{\Omega},
$$
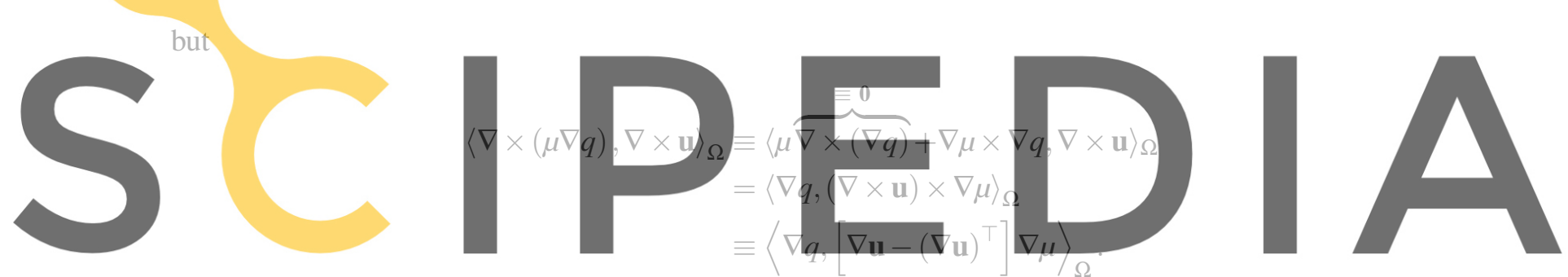

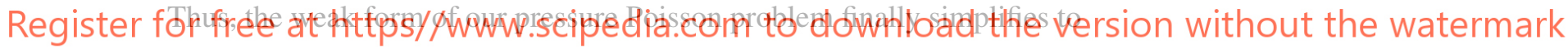

$$
\langle q, \beta \nabla \cdot \mathbf{u}\rangle_{\Omega}+\left\langle\nabla q, \nabla p+(\rho \nabla \mathbf{u}) \mathbf{u}-2(\nabla \mathbf{u})^{\top} \nabla \mu\right\rangle_{\Omega}+\langle\nabla q \times \mathbf{n}, \mu \nabla \times \mathbf{u}\rangle_{\Gamma}=0 .
$$

The last issue to be addressed is the presence of the term $\nabla \mu$ in both momentum and pressure Poisson equations. Since the viscosity in general depends on $\nabla^{s} \mathbf{u}$, computing $\nabla \mu$ in the variational formulation would increase the regularity requirements on the velocity space and therefore preclude the use of standard Lagrangian finite elements. We can avoid this by projecting the viscosity onto a continuous space, or, in other words, enforcing the rheological law weakly. Our discrete variational formulation then reads: Find $\left(\mathbf{u}_{h}, p_{h}, \mu_{h}\right) \in\left[X_{h}\right]^{d} \times X_{h} \times X_{h}$, with $\left.\mathbf{u}_{h}\right|_{\Gamma_{D}}=\mathbf{g}_{h}$, such that for all $\left(\mathbf{w}_{h}, q_{h}, v_{h}\right) \in\left[X_{h}\right]^{d} \times X_{h} \times X_{h}$, with $\left.\mathbf{w}_{h}\right|_{\Gamma_{D}}=\mathbf{0}$,

$$
\begin{aligned}
\left\langle\mathbf{w}_{h},\left(\rho \nabla \mathbf{u}_{h}\right) \mathbf{u}_{h}\right\rangle_{\Omega}+\left\langle\nabla \mathbf{w}_{h}, \mu_{h} \nabla \mathbf{u}_{h}\right\rangle_{\Omega}-\left\langle\mathbf{w}_{h},\left(\nabla \mathbf{u}_{h}\right)^{\top} \nabla \mu_{h}\right\rangle_{\Omega}-\left\langle\nabla \cdot \mathbf{w}_{h}, p_{h}\right\rangle_{\Omega} & =\left\langle\mathbf{w}_{h}, \tilde{\mathbf{t}}\right\rangle_{\Gamma_{N}}, \\
\left\langle q_{h}, \beta \nabla \cdot \mathbf{u}_{h}\right\rangle_{\Omega}+\left\langle\nabla q_{h}, \nabla p_{h}+\left(\rho \nabla \mathbf{u}_{h}\right) \mathbf{u}_{h}-2\left(\nabla \mathbf{u}_{h}\right)^{\top} \nabla \mu_{h}\right\rangle_{\Omega}+\left\langle\nabla q_{h} \times \mathbf{n}, \mu_{h} \nabla \times \mathbf{u}_{h}\right\rangle_{\Gamma} & =0, \\
\left\langle v_{h}, \mu_{h}-\eta\left(\dot{\gamma}\left(\nabla^{s} \mathbf{u}_{h}\right)\right)\right\rangle_{\Omega} & =0,
\end{aligned}
$$


with $X_{h}$ being once again a continuous finite element space. If we have a homogeneous Newtonian fluid, then $\nabla \mu \equiv \mathbf{0}$ and we recover the original boundary vorticity stabilisation [45]. We therefore denote the present method as generalised boundary vorticity stabilisation (GBVS). For optimal convergence, the function $\beta$ must be taken as the inverse of the PSPG stabilisation parameter, which for a diffusiondominated flow gives $\left.\beta\right|_{\Omega_{e}}=\left(\alpha h_{e}{ }^{2} / \mu_{h}\right)^{-1}$, resulting in

$$
\left\langle q_{h}, \beta \nabla \cdot \mathbf{u}_{h}\right\rangle_{\Omega}=\alpha^{-1} \sum_{e=1}^{N_{e}} h_{e}{ }^{-2}\left\langle q_{h}, \mu_{h} \nabla \cdot \mathbf{u}_{h}\right\rangle_{\Omega_{e}}
$$

A more detailed discussion on the choice of $\beta$ is presented by Pacheco et al. [45].

Finally, we have attained a stabilised formulation retaining full consistency even for linear elements, since all second-order derivatives have been eliminated and no part of the residual is lost. This, along with the fact that our stabilisation term is constructed from a Poisson equation with consistent pressure boundary conditions, results in a formulation that is free from spurious pressure boundary layers [45].

This will be illustrated with a numerical example.

\section{NUMERICAL EXAMPLES}

We now consider two simple benchmark tests to showcase the performance of our new stabilised formulation. Bilinear quadrilateral elements are used in both examples, with the popular Carreau-Yasuda rhelogical model
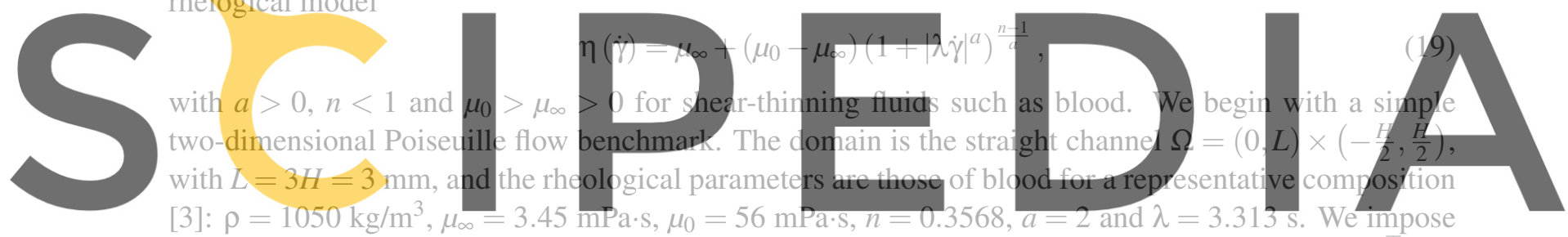

no slip on the walls, i.e., $\left.\mathbf{u}\right|_{\gamma= \pm H / 2}=0$, and enforce a 9 Pa pressure drop by setting $\left.\tilde{\mathbf{t}}\right|_{x=0}=\left\{p_{\text {in }}, 0\right\}^{\top}$ and

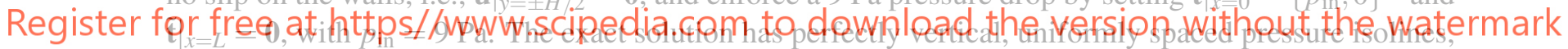

which gives an ideal scenario to investigate the issue of spurious pressure boundary layers. In order to

do that, we consider a mesh of $96 \times 32$ identical square elements and a stabilisation parameter $\alpha=20$. This is rather higher than used in practice, which allows us to really highlight the spurious effects that arise when the stabilisation term is not fully consistent. The isobars produced by the PSPG and GBVS methods are depicted in Figure 1. One immediately spots the numerical boundary layer attained by the classical method, which does not happen with the present approach. Of course, for finer meshes and smaller stabilisation parameters the difference tends to become less visible, but the spurious boundary behaviour will always be there (the thickness of the boundary layer in PSPG is actually proportional to $\sqrt{\alpha h^{2}}$ [46]). A quantitative assessment of the boundary accuracy can be found in the first study [45] where the Newtonian BVS was originally proposed.

As a second example, we choose the backward-facing step benchmark set up by Masud and Kwack [30], with the parameters $\rho=1060 \mathrm{~kg} / \mathrm{m}^{3}, \mu_{\infty}=3.45 \mathrm{mPa} \cdot \mathrm{s}, \mu_{0}=56 \mathrm{mPa} \cdot \mathrm{s}, n=0.22, \lambda=1.902 \mathrm{~s}$ and $a=1.25$. Considering the domain $\Omega=((0,12 H) \times(0,2 H)) \backslash([0,4 H] \times[0, H])$ shown in Figure 2, we prescribe a parabolic inflow at $x=0$, zero pseudo-traction on the outlet $x=12 \mathrm{H}$ and no slip on the remainder of the boundary. The Reynolds number for this example can be written as $\operatorname{Re}=\rho Q / \mu_{\infty}$, with 

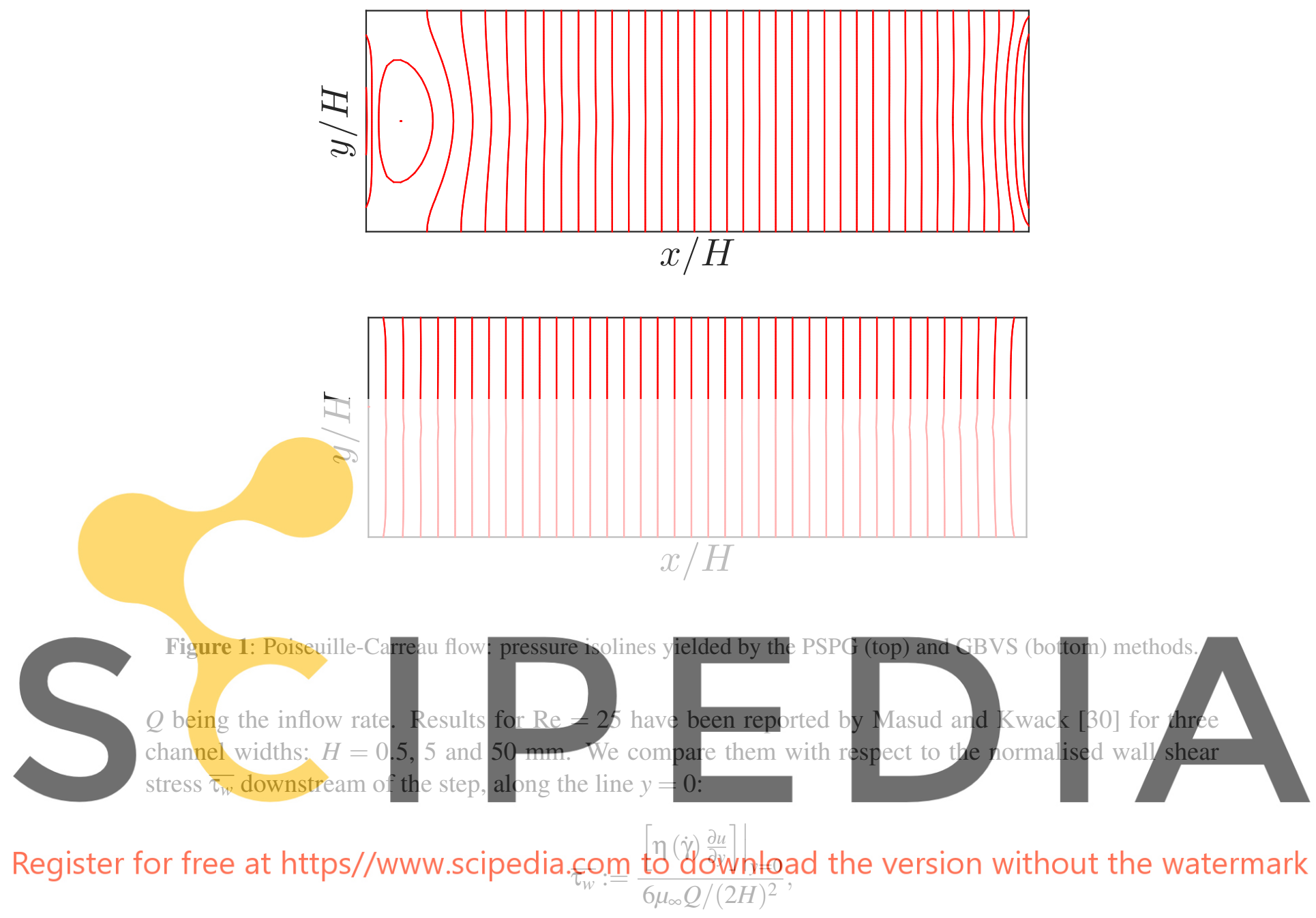

whose denominator corresponds to the wall shear stress of a developed Newtonian flow. For the numerical tests we set $\alpha=0.1$ and a uniform mesh with 50,000 identical square elements. Figure 3 shows the comparison between our results and those attained by Masud and Kwack [30] through a variational multiscale method. The comparison reveals good agreement between the solutions, especially for the narrower channels.

\section{CONCLUSIONS}

In this contribution, we have presented a new residual-based stabilisation method for equal-order finite element approximations of incompressible quasi-Newtonian flow problems. The stabilisation term is formed by combining the divergence-free constraint with a Poisson equation for the pressure with consistent boundary conditions. Furthermore, using appropriate simplifications of the nonlinear viscous term, we have managed to construct a weak formulation relying only on first-order derivatives. As a consequence, the new stabilisation term is fully consistent even for lowest-order pairs, in contrast to 


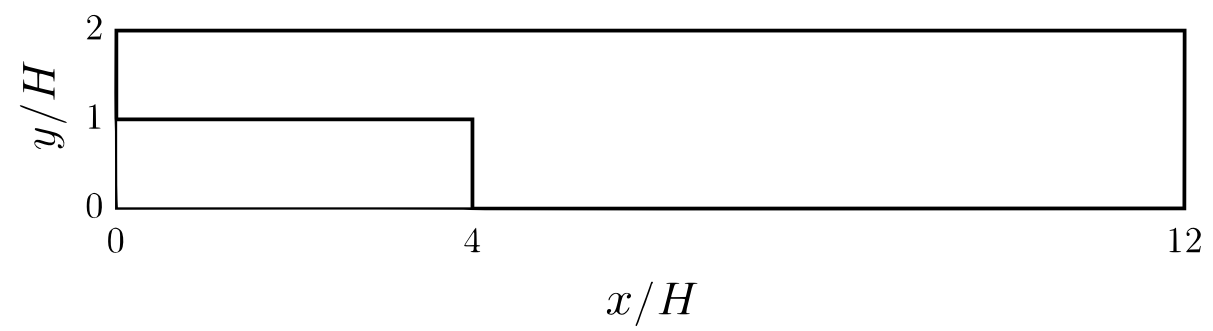

Figure 2: Geometry considered for the backward-facing step problem.

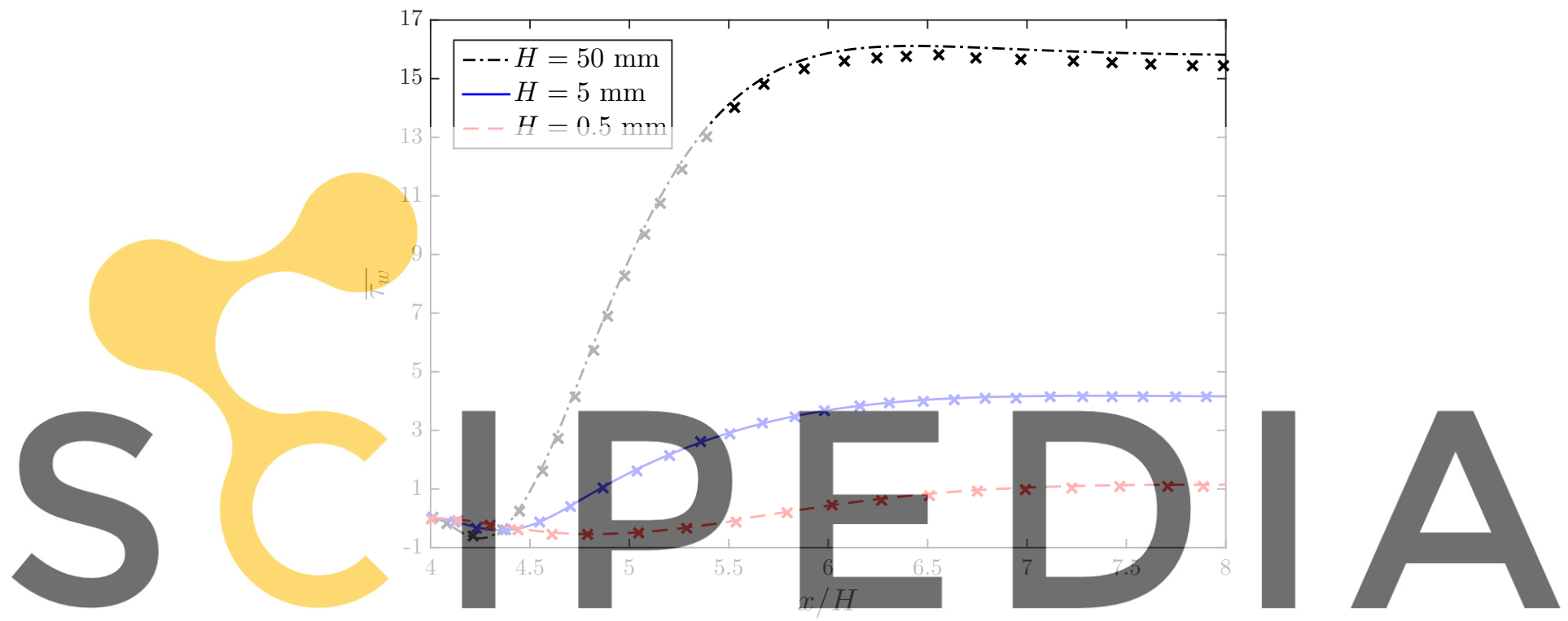

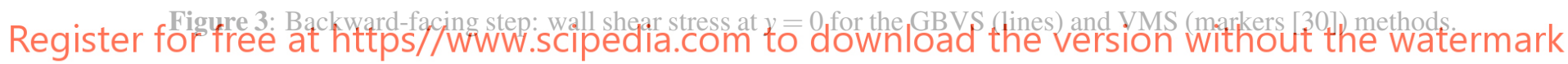

most residual-based stabilisation techniques such as PSPG, GLS and VMS. The result is a formulation completely free from spurious pressure boundary layers and improved conservation mass. As a matter of fact, numerical tests for the Newtonian case indicate improved accuracy even for higher-order finite element pairs [45]. Ongoing work includes the development of fast solvers for the time-dependent setting, including split-step and space-time schemes.

\section{ACKNOWLEDGEMENTS}

The authors acknowledge Graz University of Technology for the financial support of the Lead-project: Mechanics, Modeling and Simulation of Aortic Dissection.

\section{REFERENCES}

[1] G.P. Galdi, R. Rannacher, A.M. Robertson, and S. Turek. Hemodynamical Flows, volume 37 of Oberwolfach Seminars. Birkhäuser, Basel, 2008. 
[2] S. Chien. Shear dependence of effective cell volume as a determinant of blood viscosity. Science, 168(3934):977-979, 1970.

[3] Y.I. Cho and K.R. Kensey. Effects of the non-Newtonian viscosity of blood on flows in a diseased arterial vessel. Part 1: Steady flows. Biorheology, 28(3-4):241-62, 1991.

[4] T.J.R. Hughes, L.P. Franca, and M. Balestra. A new finite element formulation for computational fluid dynamics: V. Circumventing the Babuška-Brezzi condition: a stable Petrov-Galerkin formulation of the stokes problem accommodating equal-order interpolations. Comput. Methods Appl. Mech. Eng., 59(1):85-99, 1986.

[5] T.J.R. Hughes and L.P. Franca. A new finite element formulation for computational fluid dynamics: VII. The Stokes problem with various well-posed boundary conditions: Symmetric formulations that converge for all velocity/pressure spaces. Comput. Methods Appl. Mech. Eng., 65(1):85-96, 1987.

[6] L. Rubart and G. Böhme. Numerical simulation of shear-thinning flow problems in mixing vessels. Theor. Comput. Fluid Dyn., 3(2):95-115, 1991.

[7] H.R. Tamaddon-Jahromi, D. Ding, M.F. Webster, and P. Townsend. A Taylor-Galerkin finite element method for non-Newtonian flows. Int. J. Numer. Methods Eng., 34(3):741-757, 1992.

[8] J.P.W. Baaijens, A.A. Van Steenhoven, and J.D. Janssen. Numerical analysis of steady generalized
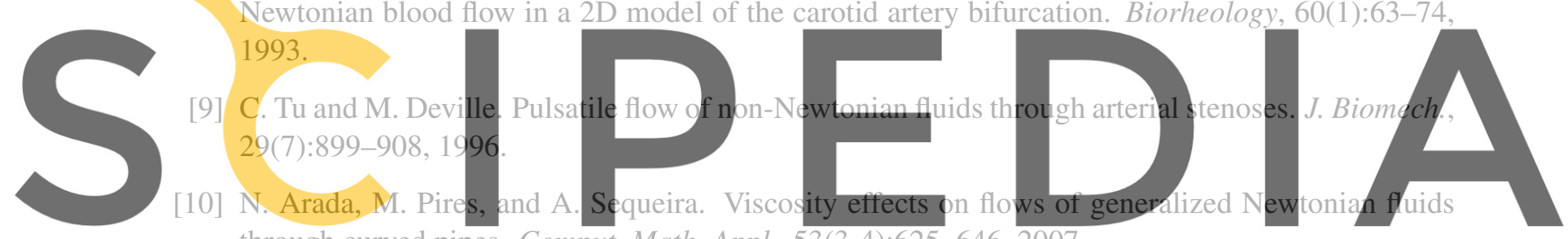
through curved pipes. Comput. Math. Appl., 53(3-4):625-646, 2007.

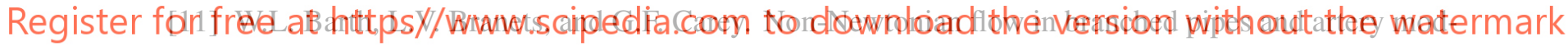
els. Int. J. Numer. Meth. Fluids, 57(5):531-553, 2008.

[12] M.G.H.M. Baltussen, Y.J. Choi, M.A. Hulsen, and P.D. Anderson. Weakly-imposed Dirichlet boundary conditions for non-Newtonian fluid flow. J. Non-Newton. Fluid Mech., 166(17-18):9931003, 2011.

[13] H. Lee. Numerical approximation of quasi-Newtonian flows by ALE-FEM. Numer. Methods Partial Differ. Equ., 28(5):1667-1695, 2012.

[14] H. Damanik, J. Hron, A. Ouazzi, and S. Turek. Monolithic Newton-multigrid solution techniques for incompressible nonlinear flow models. Int. J. Numer. Meth. Fluids, 71(2):208-222, 2013.

[15] X. Zheng, G. Chen, and X. Xie. A divergence-free weak Galerkin method for quasi-Newtonian Stokes flows. Sci. China Math., 60(8):1515-1528, 2017.

[16] J. Deteix and D. Yakoubi. Shear rate projection schemes for non-Newtonian fluids. Comput. Methods Appl. Mech. Eng., 354:620-636, 2019. 
[17] H. Sobhani, M. Razavi-Nouri, and M.H.R. Ghoreishy. Investigation of combination of finite element formulation and element type on the accuracy of 3D modeling of polymeric fluid flow in an extrusion die. J. Appl. Polym. Sci., 120(3):1607-1615, 2011.

[18] G.F. Carey, K.C. Wang, and W.D. Joubert. Performance of iterative methods for Newtonian and generalized Newtonian flows. Int. J. Numer. Meth. Fluids, 9(2):127-150, 1989.

[19] G. Böhme and L. Rubart. Non-Newtonian flow analysis by finite elements. Fluid Dyn. Res., 5(3): 147-158, 1989.

[20] M. Franta, J. Málek, and K.R. Rajagopal. On steady flows of fluids with pressure- and sheardependent viscosities. Proc. Math. Phys. Eng. Sci., 461(2055):651-670, 2005.

[21] F. Brezzi and J. Pitkäranta. On the stabilization of finite element approximations of the Stokes equations. In W. Hackbusch, editor, Efficient solutions of elliptic systems, pages 11-19. . Notes on Numerical Fluid Mechanics, 1984.

[22] S. Knauf, S. Frei, T. Richter, and R. Rannacher. Towards a complete numerical description of lubricant film dynamics in ball bearings. Comput. Mech., 53(2):239-255, 2014.

[23] C.R. Dohrmann and P.B. Bochev. A stabilized finite element method for the Stokes problem based on polynomial pressure projections. Int. J. Numer. Meth. Fluids, 46(2):183-201, 2004.

[24] L. John, P. Pustějovská, and O. Steinbach. On the influence of the wall shear stress vector form on hemodynamic indicators. Comput. Vis. Sci., 18(4-5):113-122, 2017.

[25] N.S. Hanspal, A.N. Waghode, V. Nassehi, and R.J. Wakeman. Numerical analysis of coupled Stokes/Darcy flows in industrial filtrations. Transp. Porous Media, 64(1):73-101, 2006.

[26] K. Chitra, T. Sundararajan, S. Vengadesan, and P. Nithiarasu. Non-Newtonian blood flow study in a model cavopulmonary vascular system. Int. J. Numer. Meth. Fluids, 66(3):269-283, 2011.

[27] I.G. Donev and B.D. Reddy. Time-dependent finite element simulations of a shear-thinning viscoelastic fluid with application to blood flow. Int. J. Numer. Meth. Fluids, 75(9):668-686, 2014.

[28] W. Gao and R. Liu. A hybrid finite volume/finite element method for incompressible generalized Newtonian fluid flows on unstructured triangular meshes. Acta Mech. Sin., 25(6):747-760, 2009.

[29] B. Hübner and D. Dinkler. A simultaneous solution procedure for strong interactions of generalized Newtonian fluids and viscoelastic solids at large strains. Int. J. Numer. Methods Eng., 64(7):920939, 2005.

[30] A. Masud and J. Kwack. A stabilized mixed finite element method for the incompressible shear-rate dependent non-Newtonian fluids: Variational Multiscale framework and consistent linearization. Comput. Methods Appl. Mech. Eng., 200(5-8):577-596, 2011.

[31] V.L. Marrero, J.A. Tichy, O. Sahni, and K.E. Jansen. Numerical study of purely viscous nonNewtonian flow in an abdominal aortic aneurysm. J. Biomech. Eng., 136(10), 2014.

[32] L. Gesenhues, J.J. Camata, A.M.A. Côrtes, F.A. Rochinha, and A.L.G.A. Coutinho. Finite element 
simulation of complex dense granular flows using a well-posed regularization of the $\mu(\mathrm{I})$-rheology. Comput. Fluids, 188:102-113, 2019.

[33] F. Zinani and S. Frey. Finite element approximations for quasi-Newtonian flows employing a multifield GLS method. Comput. Mech., 48(2):139-152, 2011.

[34] E. Castillo and R. Codina. Stabilized stress-velocity-pressure finite element formulations of the Navier-Stokes problem for fluids with non-linear viscosity. Comput. Methods Appl. Mech. Eng., 279:554-578, 2014.

[35] D.R.Q. Pacheco and O. Steinbach. On the pressure Poisson equation for the Stokes system. Proc. Appl. Math. Mech, 19(1), 2019. doi: 10.1002/pamm.201900425.

[36] D.R. Q. Pacheco and O. Steinbach. A continuous finite element framework for the pressure Poisson equation allowing nonNewtonian and compressible flow behavior. Int. J. Numer. Meth. Fluids, 2020. doi: 10.1002/fld.4936.

[37] V. John. Finite element methods for incompressible flow problems. Springer, New York, 2016.

[38] J.-J. Droux and T.J.R. Hughes. A boundary integral modification of the Galerkin least squares formulation for the Stokes problem. Comput. Methods Appl. Mech. Eng., 113(1-2):173-182, 1994.

[39] K.E. Jansen, S.S. Collis, C. Whiting, and F. Shaki. A better consistency for low-order stabilized finite element methods. Comput. Methods Appl. Mech. Eng., 174(1-2):153-170, 1999.

[40] E. Burman and P. Hansbo. Edge stabilization for the generalized Stokes problem: A continuous interior penalty method. Comput. Methods Appl. Mech. Eng., 195(19-22):2393-2410, 2006.

[41] P. Bochev and M. Gunzburger. An absolutely stable pressure-Poisson stabilized finite element method for the Stokes equations. SIAM J. Numer. Anal., 42(3):1189-1207, 2004.

[42] J.G. Heywood, R. Rannacher, and S. Turek. Artificial boundaries and flux and pressure conditions for the incompressible Navier-Stokes equations. Int. J. Numer. Meth. Fluids, 22(5):325-352, 1996.

[43] G. Arbia, I.E. Vignon-Clementel, T.-Y. Hsia, and J.-F. Gerbeau. Modified NavierStokes equations for the outflow boundary conditions in hemodynamics. Eur. J. Mech. B Fluids, 60:175-188, 2016.

[44] D.R.Q. Pacheco, T.S. Müller, G. Brenn, and O. Steinbach. On outflow boundary conditions in finite element simulations of non-Newtonian internal flows. Comput. Vis. Sci., accepted, 2020.

[45] D.R.Q. Pacheco, R. Schussnig, O. Steinbach, and T.P. Fries. A global residualbased stabilisation for equalorder finite element approximations of incompressible flows. Int. J. Numer. Methods Eng., 2020. doi: 10.1002/nme.6615.

[46] S. Idelsohn, M. Storti, and N. Nigro. Stability analysis of mixed finite element formulations with special mention of equal-order interpolations. Int. J. Numer. Meth. Fluids, 20(8-9):1003-1022, 1995. 